\title{
Optical properties of translucent zirconia: A review of the literature
}

\author{
Manuela-Maria Manziuc, Cristina Gasparik, Marius Negucioiu*, Mariana Constantiniuc, \\ Alexandru Burde, Ioana Vlas and Diana Dudea
}

\begin{abstract}
Translucent monolithic zirconia is the newest option of zirconia-based ceramics, which aimed to substitute the opaque classic yttria-stabilized tetragonal zirconia polycrystal (Y-TZPs) in more demanding esthetic cases.

The aim of this review was to assess the available literature regarding the optical, chemical and mechanical properties of translucent zirconia ceramics.

This systematic review was developed according to the PRISMA (Preferred Reporting Items for Systematic Review and Meta-analysis) guidelines. An electronic literature search was undertaken through Medline (National Library of Medicine) via PubMed to identify relevant articles, published in the interval 2010-2018. The search was limited to the English language publications, in vitro studies of color and microstructure of translucent zirconia material.

Yttria-stabilized tetragonal zirconia polycrystals (Y-TZPs) has excellent mechanical properties, but its intense white color and high opacity represent an esthetic limit. Cubic zirconia represents a new generation of dental ceramics with molecular structure and physical properties different from the conventional zirconia. Dental manufacturers created new formulations of this restorative material, introducing new cubic varieties of zirconia with improved optical properties. Translucent monolithic zirconia provides a new restorative option that combines strength with improved esthetics, due to its increased translucency. Translucent zirconia is indicated for anterior and posterior restorations but should be used carefully for discolored teeth, because the background color can affect the final esthetic appearance of the restoration.
\end{abstract}

Keywords: Monolithic zirconia, translucent zirconia, color, optical properties, light scattering, light transmission

Department of Prosthetic Dentistry and Dental Materials, Iuliu Hatieganu University of Medicine and Pharmacy, Cluj-Napoca, Romania

*Corresponding author: M. Negucioiu E-mail: marius.negucioiu@umfcluj.ro

DOI: 10.2478/ebtj-2019-0005
(C) 2019 Authors. This work was licensed under the Creative Commons AttributionNonCommercial-NoDerivs 4.0 License.

\section{Introduction}

Achieving a natural appearance of an artificial restoration is a demanding process, due to the complex optical characteristics of teeth (1). Over the years, metal-ceramic restorations have been considered the gold standard in dentistry, due to their increased strength and long-term longevity (2). The esthetic appearance of these restorations is influenced by the alloy used (base, noble or high noble) and its color (yellow or gray) (3), but also by the thickness, color and intrinsic translucency of layering ceramics. The esthetic outcome is also highly influenced by the marginal design; a metal-ceramic butt margin may not provide ideal light transmission, with greying or shadowing appearance at the cervical margin (4).

The esthetic requirements of dental patients have been increased overtime; as a result, a great variety of all ceramic systems have been introduced on the market $(1,2)$. Based on their chemical composition, ceramic materials can be classified into three categories: glass-matrix ceramics, polycrystalline ceramics and resin-matrix ceramics.

Glass ceramics (feldspathic, leucite-reinforced glass ceramics, lithium disilicate glass ceramics) are composed of a glass matrix containing dispersed crystalline minerals (feldspar, silica, alumina) $(5,6)$. These systems are recommended for esthetic restorations due to their increased translucency. However, these systems have limited capacities to conceal 
a discolored abutment (7). Also, their mechanical properties such as fracture toughness and flexural resistance are lower compared with those of other ceramic materials.

Glass-infiltrated ceramics are glass-matrix ceramics, composed of infiltrating molten glass with partially sintered oxides such as alumina, magnesia-alumina, and alumina-zirconia. This system is only used in combination: a core of glass-infiltrated ceramics with relatively high opacity that provides strength and allows the masking of discolored abutments, and a more translucent layering ceramics (8). The translucency of this material varies, depending on the type and amount of oxide particles; the inclusion of zirconia limits its indication as a material used in esthetic demanding cases.

Oxide ceramics are polycrystalline ceramics without a glassy phase and having all the particles densely packed. These materials are the toughest ceramics used in dentistry, their flexural strength and toughness are higher than the other ceramic systems.

Densely sintered aluminum-oxide (Procera Alumina, Nobel Biocare) was the first material introduced on the market. It has been used for anterior and posterior full ceramic restorations; it is recommended in clinical cases where average translucency is needed $(9,11)$. Another oxide ceramic is yttria-stabilized tetragonal zirconia polycrystals (Y-TZPs). Y-TZP presents superior mechanical proprieties, and it is indicated for the milling of anterior and posterior copings, full ceramic crowns, copings, and frameworks for fixed partial dentures (12-14).

Zirconia is a material that can exist in three crystalline phases: tetragonal, monoclinic and cubic (15). Pure zirconia has a cubic structure at temperatures above $2,370{ }^{\circ} \mathrm{C}(16,17)$, with the crystals in the form of a cube with square sides and possesses moderate mechanical properties $(18,19)$.

The tetragonal phase occurs at temperatures between 1,170 ${ }^{\circ} \mathrm{C}$ and $2,370{ }^{\circ} \mathrm{C}(16)$, crystals have the form of straight prisms with rectangular sides, having the best mechanical properties $(18,19)$. The monoclinic phase occurs at room temperatures to $1,170^{\circ} \mathrm{C}(16,17)$, has a deformed parallelepiped shape and possesses the weakest mechanical properties $(18,19)$. Zirconia undergoes a tetragonal to monoclinic phase transformation when cooling down from high temperatures after sintering, which makes the sintered material unstable (20). This results in a $3 \%$ to $5 \%$ expansion in the grains volume (21), which result in surface roughening, micro-cracking and deterioration of the mechanical characteristics (22-26).

However, this dimensional change of the grains creates compressive stress that inhibits crack propagation, a phenomenon that is called transformation toughening $(27,28)$. In stabilisation of the tetragonal zirconia at room temperature, to control phase transformation, different metal oxides can be added to the crystalline structure $(29,30)$.

Resin-matrix ceramics are dental ceramics composed of an organic matrix combined with ceramic fillers. The hybrid ceramic is a new category of restorative material, with a dual structure: the ceramic network structure is reinforced with a polymer network structure.

The esthetic value of a metal free restoration is based on its optimal shade and translucency with the addition of form and surface texture to mimic the natural tooth. Compared to the glass-ceramics, zirconia has a whitish opaque appearance (31), due to its poor light transmission and high reflectance.

The optical properties of teeth and dental materials include color (hue, value, and chroma), translucency, opalescence, fluorescence, iridescence. Hue is the basic color; for the tooth, it ranges in the yellow-orange tones. Saturation is the intensity of the chromatic pigment; value is the reflected light. Translucency is one of the most important factors involved in the natural aspect of restorations; it is paramount important in the selection of materials $(32,33)$; it is the ability of the material to permit the passage of the light. Highly translucent teeth or restorations tend to be lower in value; more opaque teeth or restorations, due to their increased capacity of reflecting the light, appear brighter (34).

Translucency of zirconia is dependent on: the size and shape of the constituent crystals $(35,36)$, the quantity and type of additives (37), the heating methods, temperature and atmospheric conditions used for sintering $(35,38,39)$ and the inclusion of pores which influence light scattering (40).

Translucent monolithic zirconia is the most recently developed zirconia material for anterior and posterior restorations. This material combines the strength of zirconia with improved esthetics because of its higher translucency.

\section{Purpose}

The aim of this systematic review was to gather information regarding chemical, mechanical and optical proprieties of translucent zirconia ceramic material for dental use.

The systematic review was structured according to the PRISMA (Preferred Reporting Items for Systematic Review and Meta-analysis guidelines. An electronic research was undertaken through Medline (National Library of Medicine) via PubMed to identify relevant articles. The following keywords were used: "monolithic zirconia", "translucent zirconia", "color", "optical proprieties", "light scattering", "light transmission". The search was limited to the English language; studies published in the interval 2010-2018 were included in the review.

\section{Structure and optical properties of translucent zirconia}

Various types of zirconia are available for dental applications, including partially stabilized zirconia (PSZ), tetragonal zirconia polycrystal (TZP), zirconia toughened alumina (ZTA) and fully cubic stabilized zirconia (CSZ) (41-44). The first version of conventional zirconia, one of the most used dental ceramic material (45), was the high-strength tetragonal crystalline phase, stabilized with $3 \mathrm{~mol} \%$ yttria (3Y-TZP) and enhanced with $0.25 \%$ alumina, to minimize the low temperature degradation $(45,46)$. Conventional zirconia has excellent strength, but poor translucency.

Dental manufacturers tried to satisfy the interest for higher esthetic monolithic zirconia ceramics by creating unique formulations of this restorative material (44). As a result, new translucent variants of zirconia have been developed with improved optical properties. 


\section{Methods suggested to increase the translucency of zirconia}

One of the methods of increasing zirconia translucency concerned decreasing the alumina content below $0.05 \%$, which did not influence significanly the mechanical properties of the material. However, no important increase in translucency has been generated with this process (47). Another approach of improving zirconia translucency was increasing the lanthanum oxide content to $0.2 \% \mathrm{~mol}(48)$.

Grain size and sintering temperatures influence also the translucency of zirconia (49-51). Grain size and shape is associated with a delay in low temperature degradation caused by inhibition of the transformation from the tetragonal phase to the monoclinic phase, with consequences on translucency and mechanical properties $(35,36)$.

Due to its birefringent nature, tetragonal zirconia has high opacity. The index of refraction is anisotropic in different crystallographic directions, inducing reflection and refraction phenomena at grain boundaries and reducing light transmittance (48). Therefore, a new approach to increase the translucency of zirconia was to develop an isotropic cubic zirconia material, which decreases light scattering from birefringent grain boundaries (48). This was achieved by using an increased percentage of yttria to stabilise zirconia composition, resulting in a 10 to $15 \%$ cubic crystalline zirconia phase interposed with the tetragonal phase (47).

Translucent zirconia is definitely more translucent than conventional zirconia, due to reduced grain size and increased density of the material (52). An opaque polycrystalline ceramic can be made translucent when reducing the grain sizes to sub-micron or nano-scale $(38,53)$. To decrease the scattering of the light and, by this, to gain translucency, the grain size of translucent zirconia should be less than the visible wavelength, which is in the range of 400-700 $\mathrm{nm}$. The translucency increases by maintaining zirconia grain size small, ideally under $100 \mathrm{~nm}$, and through eliminating defects such as oxygen particles and pores (48). However, extremely small grain sizes (in range of $200 \mathrm{~nm}$ ) can affect the transformation toughening mechanism of zirconia. This would produce a decrease in strength and resistance to fracture of translucent zirconia (21).

Kim et al. (54) reported that the sintering time affects the grain size of zirconia ceramic. They concluded that a short sintering time yields small grain size and increases the light transmittance values of translucent zirconia ceramic. However, the transmittance increases with the rising of the sintering temperature (from 1350 to $1550{ }^{\circ} \mathrm{C}$ ) and the zirconia polycrystalline structure becomes more compact, with a decreased porosity $(54,55)$.

To increase the translucency of zirconia, residual pores and impurities had to be decreased because they represent volumes with differences in the refractive index which led to optical scattering on the surface of zirconia, resulting in reduction of translucency $(13,56)$. Pores and pore density have a great influence on light scattering in zirconia ceramics, mainly when they are comparable in size with the wavelengths of visible light (400-700nm). Due to the typical pore size (in the range of 200-400nm) and pore density $(0,05 \%)$, conventional zirconia ceramic has a poor translucency appearance (48). The translucent zirconia shows a minimal nano-sized porosity (52).

\section{Mechanical properties of translucent zirconia}

The new translucent zirconia has a molecular structure different from a conventional zirconia, which result also in variations of the physical properties: flexural strength and fracture resistance. Cubic zirconia has lower flexural strength (ranging between 600 to $800 \mathrm{MPa}$ ) than tetragonal zirconia (which is $1000-1200 \mathrm{MPa}$ ); it can be stated that its flexural strength is higher than that of IPS e.max lithium disilicate (Ivoclar Vivadent) which is $460 \mathrm{MPa}$, but it is lower than conventional zirconia (58). However, the flexural strength of monolithic translucent zirconia restorations is higher than that of core ceramics stratified with layering porcelain (59, $60,61,62)$.

Cubic zirconia does not have the capability to perform a tetragonal to monoclinic phase transformation as tetragonal zirconia. When a fissure is initiated, tetragonal zirconia undergoes inner volumetric transformation which results to stop the crack propagation, which is not the case for cubic zirconia. The current changes in formulation that result in more esthetic appearance have not only lowered flexural strength from 1000 to $600 \mathrm{MPa}$, but also have eliminated the unique transformation toughening that gives zirconia its toughness and resistance to fracture (63). Although, the resistance to fracture of cubic zirconia is higher than that of porcelain-veneered crowns or lithium disilicate restorations $(64,65)$, this ceramic is indicated in less-bearing clinical situations (66). The manufacturers claim that the great innovation in the formula was developed at the powder stage, before discs or blocks for CAD-CAM technology were designed.

\section{Clinical characteristics for restorations with translucent zirconia}

Due to their inherent translucency, all-ceramic systems allow dentists and laboratory technicians to fabricate restorations that are similar to natural teeth. Translucency was identified as one of the primary factors in controlling esthetics $(32,33)$, and this property is critical in the selection of ceramic materials.

Due to its opacity, zirconia had as major indication the copings, which are further stratified with translucent porcelain. Compared with the coping of metal-ceramic restorations, zirconia core is whitish; the ceramic layer may be more translucent, allowing the zirconia core to show through it.

However, a common problem of ceramics layered upon a coping is the high rate of chipping. The risk of ceramic fracture and chipping was reduced by introducing the monolithic zirconia restorations. It is claimed that monolithic zirconia restorations require less tooth structure reduction in comparison with metal-ceramic. The flexural strength and fracture toughness of monolithic zirconia reduce the potential for chipping and fractures. However, the cubic zirconia is not indicated for posterior bridges with more than three units (64). Therefore, the new blocks of zirconia ceramics are indicated for creating, by CADCAM technique, anterior restorations and full-mouth rehabilitations (44). 
Configuration and optical outcome of monolithic zirconia restorations

As any modern restorations, zirconia monolithic partial or full crowns aim to improve shape, function, and color of the tooth.

It was shown that the thickness of the restoration affects the light transmission through ceramics. By reducing the thickness of the restoration, the material would be more translucent and natural-looking, but less fracture resistant. On the contrary, by increasing the thickness, the esthetic and the translucency would be decreased, but the strength and resistance to stress would be increased (67).

Monolithic zirconia restorations can be used as an alternative to conventionally veneered zirconia, due to their increased mechanical properties even at a minimal thickness $(60,68,69,70)$. For clinical use, the thickness for conventional monolithic zirconia restorations could be considered $0,5-0.75 \mathrm{~mm}$. The new translucency zirconia exhibits favorable mechanical properties and esthetic performance at $0,5-1 \mathrm{~mm}$ (67).

Conventional zirconia has excellent strength, but its color is white and opaque as chalk; to individualize the color and translucency of a natural tooth, it is required important post-milling work, like stratification of feldspathic porcelain. The introducing of translucent zirconia blocks, which are directly milled into monolithic restorations has been one of the most challenging innovation of the last decade in dentistry.

In addition, the translucent zirconia discs are provided in a high range of colors, which reduces the need of surface stains. Translucent zirconia discs have been developed beyond simply achieving optimal translucency and color that match the VITA Classical Shade Guide. Initially, even high-translucency zirconia restorations were stained and glazed after milling to gain a natural appearance for the frontal teeth; more recently, preshaded, multilayer and high-translucent zirconia discs have been developed, to mimic the gradient in color and translucency along the dental crown from the incisal to the cervical portion, as well as between the dentin and the enamel layers. In addition, customised staining and extrinsic characterisation can be used (44).

For the full ceramic restorations, it is important to take also into account the color of the tooth to be restored; in the case of a dichromic condition, the material has to mask the dark substrate. In this respect, the teeth without discoloration are better restored with a more translucent zirconia, while dichromic teeth would need a more opaque zirconia ceramic restoration.

High translucency zirconia is indicated for thin, limited restorations as laminate veneers and partial crowns. This material might be appropriate for replacing enamel, without a non-necessary increase in dental volume. However, too much translucency must be avoided because the restoration might appear greyish (71).

Medium translucency zirconia is indicated for dentin replacement but is not recommended for enamel replacement, and the veneering process will be necessary for a good aesthetic result (72).

Low translucency zirconia might be indicated as a core material for dentin replacement. The high opacity zirconia might be indicated to mask the underlying abutment, like discolored teeth, metal post or metallic abutment.

Due to its translucency, the esthetic appearance of translucent zirconia restoration can be affected by the discromic background. But, the final color of a translucent restoration is also affected by the luting cement $(72,73)$. However, the thickness of translucent zirconia restoration and cement shade has an obvious effect on the final color.

\section{Configuration of the abutments for monolithic zirconia restorations}

All ceramic restorations have an optimal aesthetic appearance when tooth preparation is accurately performed. This involves a minimally invasive preparation, with the highest preservation of the remaining natural dental structure. For conventional all-ceramic restorations, the tooth reduction for the occlusal clearance would be $2 \mathrm{~mm}$ and for proximal and axial clearance $1,5 \mathrm{~mm}$ (74). However, for the cubic monolithic zirconia restorations a clearance of $0,5-1 \mathrm{~mm}$ is needed, according to a specific clinical situation.

The finish line design for a monolithic zirconia restorations is rounded shoulder or light chamfer of $0,5 \mathrm{~mm}$ width (75). The framework design has a great influence on fracture resistance of a translucent monolithic zirconia bridge. Therefore, to obtain the same resistance to fracture, the connector area of two translucent zirconia crowns should measure $9 \mathrm{~mm}^{2}$, compared to the connector area of lithium disilicate restorations of 16 $\mathrm{mm}^{2}(76)$.

\section{Polishing and glazing of monolithic zirconia restorations}

The surface roughness of monolithic zirconia restorations could cause the wear on enamel of antagonists. Mitov et al reported in their study that rough monolithic zirconia restorations produce more wear of the antagonist enamel than polished monolithic crowns (77).

Stawarczyk et al found in their study that glazed monolithic translucent zirconia restoration presents the most abrasive ceramic surface for antagonist enamel, while the polished zirconia is the least abrasive (78). Mundhe et al have shown that polished zirconia restorations cause less tooth structure wear of the opposing enamel than feldspathic ceramics (79). Janyavula et al compared in their study the roughness of polished, glazed, polished and glazed veneered monolithic zirconia restorations (80). They conclude that polished zirconia had the smoothest ceramic surface.

However, other studies showed that polished translucent zirconia restorations present very smooth surface. Therefore, the ceramic surface of these dental materials is less abrasive when compared with other ceramic systems, like lithium disilicate (81).

In some clinical situations, after CAD/CAM milling, the monolithic restoration has the basic contours, without a natural texture. For achieving the most natural look, texturizing can be performed by the dental technician using a specific protocol. McLaren showed in his study that only by glazing the translucent zirconia it can not be achieved a natural look of the res- 
toration (82). He suggested that polishing procedures provide a more natural, realistic appearance, compared to glazed ones. Nevertheless, the are clinical situations, when for achieving a more natural look, surface staining pigments can be used to create individualized characterisation (76).

After delivery in dental office and performing the try-in procedures, some adjustment of monolithic zirconia restorations may be needed. The clinical occlusal adjustment can be done by using a conventional diamond bur or a special instrument with diamond grit, under water cooling. However, dental manufacturers suggest performing the crowns occlusal adjustment or contour modifications, before the sintering process, in order to achieve the desired natural appearance of the restorations and to avoid microroughness (82).

\section{Cementation of monolithic zirconia restorations}

Resin cements are the most used dental materials for cementation of full ceramic restorations, because they have good esthetics, low solubility, high strength and mechanical resistance. Dual-cure resin cements with phosphate or carboxylate groups are the best choice for bonding zirconia restorations. To increase the bond strength, the zirconia crowns are sandblasted, usually with oxide aluminium particles (64).

Lawson et al concluded in their study that sandblasting the cubic zirconia restorations with alumina particles, does not weak the material, and it is recommended for an increased bond strength. He also suggested not to sandblast the zirconia crowns when they are cemented with resin-modified glass ionomer cement (82).

Blatz et al related that resin bonding cementation increases the strength of ceramic materials and it is highly indicated, to avoiding fractures, when bonding very thin translucent zirconia restorations. He also suggested using a zirconia primer for increasing the bond strength (83).

However, due to translucency of translucent zirconia restoration, the final color can be affected by the shade of the luting cement. Try-in paste cement can be very helpful when choosing the shade of luting resign cement (84). Considering the increased esthetic demand of patients and dentists, choosing the right shade of dental ceramic materials and luting cements is an important process.

\section{Conclusions}

Within the limitations of the present study, the literature review showed that several factors can affect the properties of a restoration made of translucent zirconia and the following conclusions were drawn:

Translucent zirconia has a molecular structure and physical properties different from the conventional zirconia; it exhibits a decreased strength and resistance to stress. The optical and mechanical properties are influenced by factors such as grains and pores size and configuration of the crystals.

This material exhibits mechanical and esthetic properties in between the conventional zirconia and lithium disilicate. The preshaded and multilayered translucent zirconia blocks provide mechanical properties and good esthetics, being appropriate to restore individual anterior teeth, but also in the construction of full-mouth rehabilitations.

Polished translucent zirconia restorations cause less wear tooth structure of antagonist enamel than glazed ones or other ceramic materials.

The esthetic appearance of translucent zirconia restoration can be affected by the discolored background, and the luting cement. The thickness of translucent zirconia, as well as the cement shade and translucency has an important effect on the final color of the restoration.

\section{Acknowledgements}

This article is part of a project (1300/39/13.01.2017) supported by the University of Medicine and Pharmacy "Iuliu Hatieganu" Cluj-Napoca, Romania.

\section{Conflict of interest statement}

The authors declare no financial or commercial conflict of interest.

\section{References}

1. Vichi A, Louca C, Corciolani G, Ferrari M. Color related to ceramic and zirconia restorations: A review. Dental Materials 2011; 27: 97108.

2. Raptis NV, Michalakis KX, Hirayama H. Optical behaviour of current ceramic systems. International Journal of Periodontics and Restorative Dentistry 2006; 26: 31-41.

3. O'Boyle KH, Norling BK, Cagna DR, Phoenix RD. An investigation of new metal framework design for metal ceramic restorations. J Prosthet Dent 1997; 78: 295-301.

4. Christensen GJ. Choosing an all-ceramic restorative material: porcelain-fused-to-metal or zirconia based? J Am Dent Assoc 2007; 138: 662-5.

5. Touati B, Miara P, Nathanson D. Esthetic Dentistry and Ceramic Restorations. London:Marin Dunitz, 1999.

6. Anusavice KJ(ed). Philip's Science of Dental Materials, ed 10. Philadelphia: WB Saunders, 1996.

7. Cohen M. Interdisciplinary treatment planing.Quintessence Publishing Co; 2008: 383-406.

8. Heffernan MJ, Aquilino SA, Diaz-Arnold AM, et al. Relative translucency of six all-ceramic systems. Part I: Core materials. J Prosthet Dent 2002; 88: 4-9.

9. Oden A, Andersson M, Krystek-Ondracek I, Magnusson D. Fiveyear clinical evaluation of Procera AllCeram crowns. J Prosthet Dent 1998; 80: 450-6.

10. Odman P, Andersson B. Procera AllCeram crowns followed for 5 to 10 years: A prospective clinical study. Int J Prosthodont 2001; 14: 504-9.

11. Fradeani M, D’Amelio M, Redemagni M, Corrado M. Five-year follow-up with Procera all-ceramic crowns. Quintessence Int 2005; 36: 105-13.

12. Christel P, Meunier A, Heller M. Mechanical properties and short term in-vivo evaluation of yttrium-oxide-partially-stabilized zirconia. J Biomed Mater Res 1989; 23: 45-61.

13. Piconic C, Macauro G. Zirconia as a ceramic biomaterial. Biomaterials 1999; 20: 1-25.

14. Raigrodski AJ. Contemporary materials and technologies for all-ceramic fixed partial dentures: A review of the literature. J Prosthet Dent 2004; 92: 557-62.

15. Howard CJ, Hill RJ. The polymorphs of zirconia: phase abundance and crystal structure by Rietveld analysis of neutron and X-ray diffraction data. J Mater Sci. 1991; 26(1): 127-34. 
16. Volpato CAM, Garbelotto LG, Fredel MC, et al. Application of zirconia in dentistry: Biological, mechanical and optical considerations. In: Sikalidis C (ed). Advances in Ceramics: Electric and Magnetic Ceramics, Bioceramics, Ceramics and Environment. New York: InTech, 2011.

17. Guazzato M, Quach L, Albakry M, Swain MV. Influence of surface and heat treatments on the flexural strength of Y-TZP dental ceramic. J Dent 2005; 33: 9-18.

18. Kingery WD, Bowen HK, Uhlmann DR. Introduction to Ceramics, ed.2. New York: John Wiley, 1976.

19. Helvey GA. Zirconia and computer-aided design/computer-aided manufacturing (CAD/CAM) dentistry. Funct Esthet Restorative Dent 2007; 1: 28-39.

20. Yanagida H, Kawamoto K, Miyayama M. Chemistry of ceramics. Chichester, England:Wiley,1996: 226-228; 247-9.

21. Denry I, Kelly JR. State of the art of zirconia for dental applications. Dent Mater. 2008; 24(3): 299-307.

22. Roy ME, Whiteside LA, Katerberg BJ, Steiger JA. Phase transformation, roughness and microhardness of artificially aged yttria- and magnesia- stabilized zirconia femoral heads. J Biomed Mater Res A 2007; 83: 1096-102.

23. Chevalier J, Gremillard L, Virkar A, Clarke DR. The tetragonal-monoclinic transformation in zirconia: lessons learned and future trends. J Am Ceram Soc 2009; 92: 1901-20.

24. Cattani-Lorente M, Scherrer SS, Ammann P, Jobin M, Wiskott HW Low-temperature degradation of a Y-TZP dental ceramic. Acta Biomater 2011; 7: 858-65.

25. Kohorst P, Borchers L, Strempel J, Stiesch M, Hassel T, Bach FW, et al. Low-temperature degradation of different zirconia ceramics for dental applications. Acta Biomater 2012; 8: 1213-20.

26. Nakamura K, Harada A, Kanno T, Inagaki R, Niwano Y, Milleding P, et al. The influence of low -temperature degradation and cyclic loading on the fracture resistance of monolithic zirconia molar crowns. J Mech Behav Biomed Mater 2015; 47: 49-56.

27. Sorenson JA. The Lava all-ceramic system: CAD/CAM zirconium. Prosthodontics for the 21st century. Synergy in Dentistry 2003; 2(suppl to Contemp Esthet Restor Pract): 3-6.

28. Hannink RHJ, Kelly PM, Muddle BC. Transformation toughening in zirconia-containing ceramics. J Am Ceram Soc 2000; 83: 461-87.

29. McLaren EA, Giordano RA. Zirconia-based ceramics: Material properties, aesthetic and layering techniques of new veneering porcelain, VM9. Quintessence Dent Technol 2005; 28: 100.

30. Tanaka K, Tamura J, Kawanabe K, et al. Ce-TZP/A1203 nanocomposite as a bearing material in total joint replacement. J Biomed Mater Res 2002; 63: 262-70.

31. Chen YM, Smales RJ, Yip KH, Sung WJ. Translucency and biaxial flexural strength of four ceramic core materials. Dent Mater 2008; 24: 1506-11.

32. Kelly JR, Nishimura I, Campbell SD. Ceramics in dentistry: historical roots and current perspectives. J Prosthet Dent 1996; 75: 18-32.

33. Watts DC, Cash AJ. Analysis of optical transmission by 400-500 nm visible light into aesthetic dental biomaterials. J Dent 1994; 22: 112 7.

34. Galip Gurel. The Science and Art of Porcelain Laminate Veneers. Quintessence Publishing. Germany, 2003, pp.159-204.

35. Yang D, Raj R, Conrad H. Enhanced sintering rate of Zirconia (3Y-TZP) through the effect of a weak dc electric field on grain growth. J Am Ceram Soc 2010; 93: 2935-7.

36. Li JF, Watanabe R. Phase Transformation in Y2O3-Partially-Stabilized ZrO2 Polycrystals of Various Grain Sizes during Low-Temperature Aging in Water. J Am Ceram Soc 1998; 81: 2687-91.

37. Casolco SR, Xu J, Garay JE. Transparent/translucent polycrystalline nanostructure yttrium stabilized zirconia with varying colors. Scr Mater 2008; 58: 516-9.

38. Jiang L, Liao Y, Wan Q, Li W. Effects of sintering temperature and particle size on the translucency of zirconium dioxide dental ceramic. J Mater Sci 2011; 22: 2429-35.

39. Anselmi-Tamburini U, Woolman JN, Munir ZA. Transparent nanometric cubic and tetragonal zirconia obtained by high-pressure pulsed electric current sintering. Adv Funct Mater 2007; 17: $3267-$ 73.

40. Alaniz JE, Perez-Gutierrez FG, Aguilar G, Garay JE. Optical proprieties of transparent nano crystalline yttrium stabilised zirconia. Opt Mater 2009; 32: 62-8.

41. Wang H, Aboushelib MN, Feilzer AJ. Strength influencing variables on CAD/CAM zirconia frameworks. Dent Mater 2008; 24: 633-8.

42. Heuer AH, Claussen N, Krisen WM, Ruhle M. Stability of tetragonal ZrO2 particles in ceramic matrices. J Am Ceram Soc 1982; 65: 64250.

43. Zhao M, Sun J, Zhang J, Zhang Y. Novel translucent and strong sub micron alumina ceramics for dental restorations. J Dent Res 2017; $1-7$.

44. Mazda J. Shining a light on translucent zirconia. Inside Dentistry 2017; Volume 13, Issue 8 .

45. Tong H, Tanaka CB, Kaizer MR, Zhang Y. Characterization of three commercial Y-TZP ceramics produced for their high-translucency, high-strength and high-surface area. Ceram Int. 2016; 42(1 Pt B): 1077-85.

46. Matsui K, Ohmichi N, Ohgai M, et al. Effect of alumina-doping on grain boundary segregation induced phase transformation in yttria-stabilized tetragonal zirconia polycrystal. J Mater Res 2006; 21(9): 2278-89.

47. Harada K, Raigrodski AJ, Chung KH, et al. A comparative evaluation of the translucency of zirconias and lithium disilicate for monolithic restorations. J Prosthet Dent 2016; 116(2): 257-63.

48. Zhang Y. Making yttria-stabilized tetragonal zirconia translucent. Dent Mater 2014; 30(10): 1195-203.

49. Wang SF, Zhang J, Luo DW, Gu F, Tang DN, Dong ZL, et al. Transparent ceramics: processing materials and applications. Prog Solid State Chem 2013; 41: 20-54.

50. Krell A, Hutzler T, Klimke J. Transparent ceramics: transmission physics and consequences for materials selection, manufacturing and applications. J Eur Ceram Soc 2009; 29: 207-21.

51. Callister WDJR. Materials science and engineering: an introduction. 8th ed. New York: John Wiley \& Sons, Inc; 2007. p. 975.

52. Harianawala HH, Kheur MG, Apte SK, Kale BB, Sethi TS, Kheur SM. Comparative analysis of transmittance for different types of commercially available zirconia and lithium disilicate materials. J Adv Prosthodont 2014 ;6: 456-61.

53. Wang Y, Huang H, Gao L, Zhang F. Investigation of a new 3Y-stabilized zirconia with an improved optical property for applications as a dental ceramic. J Ceram Process Res 2011; 12: 473-476.

54. Kim MJ, Ahn JS, Kim JH, Kim HY, Kim WC. Effects of the sintering conditions of dental zirconia ceramics on the grain size and translucency. J Adv Prosthodont 2013; 5: 161-6.

55. Zhang HB, Kim BN, Morita K, Yoshida H, Lim JH, Hiraga K. Optimization of high-pressure sintering of transparent zirconia with nano-sized grains. J Alloy Compd. 2010; 508: 196-199.

56. Lucas TJ, Lawson NC, Janowski GM, Burgess JO. Effect of grain size on the monoclinic transformation, hardness, roughness, and modulus of aged stabilized zirconia. Dent Mater 2015; 31: 1487-92.

57. Ivoclar Vivadent. IPS e.max Lithium Disilicate. Ivoclar Vivadent website. http://www.ivoclarvivadent.com/en/p/all/products/all-ceramics/ips-emax-dentist/ips-emax-lithium-disilicate. Accessed June 23, 2017.

58. Shop and Compare: Millable Materials. Inside Dental Technology. 2017; 7(11).

59. Johansson C, Kmet G, Rivera J, et al. Fracture strength of monolithic all-ceramic crowns made of high translucent yttrium oxide- stabilized zirconium dioxide compared to porcelain-veneered crowns 
and lithium disilicate crowns. Acta Odontol Scand 2014;72:145-153.

60. Beuer F, Stimmelmayr M, Gueth JF, et al. In vitro performance of full-contour zirconia single crowns. Dent Mater 2012;28:449- 456.

61. Holand W, Schweiger M, Watzke R, et al. Ceramics as biomaterials for dental restoration. Expert Rev Med Devices 2008;5:729- 745.

62. Fischer J, Stawarczyk B and Hammerle CH. Flexural strength of veneering ceramics for zirconia. J Dent 2008; 36: 316-321.

63. Glidewell Laboratories. About Us. Glidewell Laboratories website. http://glidewelldental.com/about-us/. Accessed June 23, 2017.

64. Kwon SJ, Lawson NC, McLaren EE, et al. Comparison of the mechanical properties of translucent zirconia and lithium disilicate. J Prosthet Dent 2018

65. Zesewitz TF, Knauber AW and Northdurft FP. Fracture resistance of a selection of full-contour all-ceramic crowns: an in vitro study. Int J Prosthodont 2014; 27: 264-266.

66. Shahmiri R, Standard OC, Hart JN, et al. Optical properties of zirconia ceramics for esthetic dental restorations: A systematic review. J Prosthet Dent 2018;119:36-46.

67. Vichi A, Sedda M, Fonzar RF, Carrabba M, Ferrari M. Comparison of contrast ratio, translucency parameter, and flexural strength of traditional and "augmented translucency" zirconia for CEREC CAD/CAM System. J Esthet Restor Dent 2016; 28: 32-9.

68. Zesewitz T, Knauber W, Nothdurft FP. Fracture resistance of a selection of full-contour all-ceramic crowns: an in vitro study. Int J Prosthodont 2014; 27: 264-6.

69. Preis V, Behr M, Hahnel S, Handel G, Rosentritt M. In vitro failure and fracture resistance of veneered and full-contour zirconia restorations. J Dent 2012; 40: 921-8.

70. Sun T, Zhou S, Lai R, Liu R, Ma S, Zhou Z, et al. Load-bearing capacity and the recommended thickness of dental monolithic zirconia single crowns. J Mech Behav Biomed Mater 2014; 35: 93-101.

71. Corciolani G, Vichi A, Louca C, Ferrari M. Influence of layering thickness on the color parameters of a ceramic system. dent Mater 2010; 26(8): 737-42.

72. Vichi A, Carrabba M, Paravina R, Ferrari M. Translucency of ceramic materials for CEREC CAD/CAM System. J Esthet Restor Dent 2014; 26(4): 224-31.
73. Karaagaclioglu L, Yilmaz B. Influence of cement shade and water storage on the final color of leucite-reinforced ceramics. Oper Dent 2008; 33: 386-91.

74. Milleding P. Preparation design for traditional fixed fullcrown restorations. Preparations For Fixed Prosthodontics. Denmark: Munksgaard, 2012

75. Nakamura K, Harada A, Inagaki R, et al. Fracture resistance of monolithic zirconia molar crowns with reduced thickness. Acta Odontol Scand 2015;73:602-608

76. Rinke $\mathrm{S}$ and Fischer C. Range of indications for translucent zirconia modifications: clinical and technical aspects. Quintessence Int 2013;44:557-566.

77. Mitov G, Heintze SD, Walz S, et al. Wear behavior of dental Y-TZP ceramic against natural enamel after different finishing procedures. Dent Mater 2012;28:909-918.

78. Stawarczyk B, Ozcan M, Schmutz F, et al. Two-body wear of monolithic, veneered and glazed zirconia and their corresponding enamel antagonists. Acta Odontol Scand 2013; 71: 102-112.

79. Mundhe K, Jain V, Pruthi G, et al. Clinical study to evaluate the wear of natural enamel antagonist to zirconia and metal ceramic crowns. J Prosthet Dent. 2015;114:358-363.

80. Janyavula S, Lawson N, Cakir D, et al. The wear of polished and glazed zirconia against enamel. J Prosthet Dent 2013;109:22-29.

81. Rosentritt M, Preis V, Behr M, et al. Two-body wear of dental porcelain and substructure oxide ceramics. Clin Oral Investig 2012;16:935-943.

82. McLaren EA, Lawson N, Choi J, et al. New High-Translucent Cubic-Phase-Containing Zirconia: Clinical and Laboratory Considerations and the Effect of Air Abrasion on Strength. Compend Contin Educ Dent 2017;38:e13-e16.

83. Blatz MB, Alvarez M, Sawyer K, Brindis M. How to bond zirconia: the APC concept. Compend Contin Educ Dent. 2016;37(10):611620 .

84. Ghodsi S, Jafarian Z. A review on translucent zirconia. Eur. J. Prosthodont. Restor. Dent. 2018;26:62-74. 